\title{
Experiments in Selective Overhearing of Hierarchical Organizations $^{\star}$
}

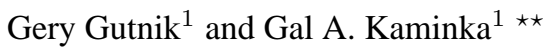 \\ The MAVERICK Group, \\ Computer Science Department, Bar-Ilan University, Israel \\ gutnikg,galk@cs.biu.ac.il
}

\begin{abstract}
Lately, overhearing has gained interest in monitoring multi-agent settings. Previous investigations provided an extensive set of techniques using overhearing. However, most previous investigations rely on a problematic assumption that all inter-agent communications can be overheard. In the real-world settings, it is reasonable to assume that the available overhearing resources will be essentially limited. Thus, overhearing targets should be carefully chosen. We provide a theoretical and empirical study of selective overhearing. In particular, we focus on overhearing hierarchical organizations that are highly popular in the real-world settings. This paper first presents a theoretical approach for modelling overhearing in hierarchical organizations. Then, based on the proposed model, we present experiments in simulating conversations in hierarchical organizations, and empirically examine a set of overhearing strategies particularly suited for such organizations. Based on these extensive experiments, we are able to determine efficient overhearing strategies and isolate the parameters influencing their behavior.
\end{abstract}

\section{Introduction}

Recent multi-agent systems (MAS) are often built applying an open, distributed architecture. These systems involve various challenges of monitoring geographicallydistributed and independently-built multiple agents. Monitoring by overhearing [1] has been found to provide a powerful monitoring technique particularly suited for open distributed MAS settings. According to this technique, an overhearing agent cooperatively monitors the exchanged communications between application agents. The overhearing agent uses these observed communications to independently assemble and infer the monitoring information on the corresponding MAS settings.

Previous investigations on overhearing have demonstrated a range of overhearing techniques. Novick and Ward [2] modelled overhearing by pilots that seek to maintain their own situational awareness. Kaminka et al. [1] developed a plan-recognition approach to overhearing in order to monitor the state of distributed agent teams. Aielo et al. [3] and Bussetta et al. [4,5] investigated an architecture that enables overhearing, so that domain experts can provide advice to problem-solving agents when necessary. Legras [6] examined the use of overhearing for maintaining organizational awareness.

\footnotetext{
* This research was supported in part by BSF Grant \#2002401.

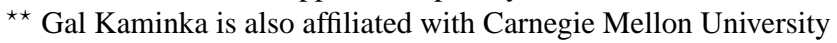


Rossi and Busetta [7] applied overhearing to monitor state transitions in multi-agent settings and recognize changes in agents' social roles. Recent investigations proposed a formal approach to overhearing: our work in [8] introduced a theoretical model to overhearing and applied it for conversation recognition, while Platon et al. [9] addressed design patterns for overhearing.

Although these previous investigations provided an extensive set of overhearing techniques, most rely on the ability of an overhearing agent to overhear all inter-agent communications. However, this assumption can be challenged both in the real-world settings and in the majority of multi-agent applications (particularly, large-scale MAS). Instead, we can reasonably assume that overhearing resources are essentially limited. Under the restriction of limited overhearing resources, a single overhearing agent or a team of overhearers will be able to overhear only a subset of conversations committed in monitored organizations. Consequently, efficient allocation of overhearing resources, i.e. selectivity in which agents will be overheard, is an important aspect of overhearing.

We propose a theoretical and empirical study of limited-resource overhearing in hierarchical organizations. These hierarchical organizational-structures are often associated with corporate and military organizations, which are widely spread in the realworld settings. This paper presents a model for overhearing hierarchical organizations providing a definition for (i) specification of conversations' characteristics in such organizations and (ii) overhearing strategies suitable for these settings. Based on the proposed model, we performed an extensive set of experiments simulating overhearing in pyramidal-hierarchical organizations. In these experiments, various centralistic overhearing strategies have been examined qualitatively and quantitatively.

Specifically, two overhearing strategies have been found to be efficient. The efficiency of overhearing strategies was measured as a percentage of optimal overhearing strategy, which can be calculated post factum (see Section 3.2). The first overhearing strategy addresses overhearing most important agents in multi-agent settings. This overhearing strategy assumes overhearing highly-valuable communications to be the key to efficiency. On the other hand, the second overhearing strategy proposes to overhear agents that are less-important, but highly-communicative (i.e. involved in a large number of conversations). Here, its efficiency is based on gathering large amounts of information.

Analyzing these strategies in various configurations, we come to some interesting conclusions. The first overhearing strategy behaves as a parabolic curve with a long tail as communication activity level increases, while the second strategy maintains its linearity. Therefore, monitoring organizations, in some conditions, it is more efficient to overhear few highly-valuable conversations, while in other conditions, it is more efficient to overhear many less-valuable conversations and, thus, gather information on monitored settings due to the quantity of overheard information. In our experiments, we have also been able to examine the various factors that influence the shape and performance of these two strategies.

This paper is organized as follows. The next section presents a brief discussion of previous investigations providing the initial motivation for our work. In Section 3, we discuss in details the proposed model for overhearing hierarchical organizations, 
whereas Section 4 describes the set of performed experiments and their results. Section 5 concludes and presents directions for future research.

\section{Background \& Motivation}

Work by Nowick and Ward [2] shows an early use of cooperative overhearing to model interactions between pilots and air-traffic controllers. In this model, pilots maintain mutuality of information with the controller not only by dialogue, but also by listening to the conversations of other pilots. While each pilot and controller act cooperatively, the other pilots are not necessarily collaborating on a joint task. Rather, they use overhearing to maintain their situational awareness out of their own self-interest. Similarly, Legras [6] uses overhearing as a method that allows agents to maintain organizational knowledge. In this approach, agents broadcast changes in their organizational memberships. Other agents use this information to maintain organizational awareness.

In contrast, investigations in [3-5] describe collaborative settings in which the overhearing agent may act on overheard messages to assist the communicating agents. The settings they describe involve communicating agents, who are engaged in problem solving. An overhearing agent monitors their conversations, and offers expert assistance if necessary.

Kaminka et al. [1] used plan recognition in overhearing a distributed team of agents, which are collaborating to carry out a specific task. Knowing the plan of this task and its steps, the monitor uses overheard messages as clues for inferring the state of different team-members. The authors presented a scalable probabilistic representation (together with associated algorithms) supporting such inference, and showed that knowledge of the conversations that take place facilitates a significant boost in accuracy.

Rossi and Busetta [7] applied overhearing to identify social roles in multi-agent settings. Initially, the authors used overhearing to monitor changes in MAS settings caused by transition from one state to another. Using a set of predefined transition rules, the monitor relies on overheard messages to follow the progress of MAS application and to determine possible faults and inconsistency. Then, the information gathered from overhearing is used to identify agent's social roles. These social roles may change over time. Thus, the monitor uses overheard messages, together with its knowledge of MAS status, to determine social roles of communicating agents at various time intervals based on a predefined set of social rules.

Finally investigations in $[8,9]$ proposed a formal approach to overhearing. Our work in [8] introduced a comprehensive theoretical model for overhearing and applied it to one of the key steps in overhearing-conversation recognition, i.e. identifying the conversation that took place given a set of overheard messages. We developed a family of algorithms to this problem and showed their relative appropriateness for large-scale settings by analyzing their complexity. Platon et al. [9] addressed overhearing in terms of its architecture and implementation. Here, overhearing is referred as a design pattern and its various types are distinguished. In addition, the authors propose a set of implementation methods for overhearing and compare their relative strengths and weaknesses. 
Most previous work assumes that all inter-agent communications can be overheard. However, this assumption is challenged in the real-world settings and in particular in large-scale multi-agent systems. In such settings, it is reasonable to assume that the overhearing resources are essentially limited. Therefore, it is important to be efficient, i.e. selective about which agents will be overheard, while others will not.

We focus on selective overhearing of organizations with hierarchical structure. These organizations are highly popular in real-world settings (e.g. corporate and military organizations). In such organizations, the importance of conversations varies with respect to organizational roles of their participants. Thus, our initial overhearing strategy has been to overhear agents with most important organizational roles. However, as we show later in the paper, in some conditions, this overhearing strategy performs poorly.

Therefore, the strategy of overhearing agents of most important organizational roles is insufficient. In this paper, we empirically determine a set of overhearing strategies that can be applied to efficiently overhear hierarchical organizations under the restriction of limited overhearing resources.

\section{Overhearing in Hierarchical Organizations}

Overhearing extracts information from conversation systems [8]-the set of conversations generated by an organization. Thus, conversation systems change based on the type of organization that is being overheard, and, in turn, overhearing agents must adapt their overhearing strategies to match the conversation system.

This section first describes the conversation systems expected to be generated in hierarchical organizations (Section 3.1). It then continues by proposing a number of overhearing strategies for such organizations (Section 3.2).

\subsection{Modelling Conversation Systems}

We define a conversation system of hierarchical organizations as a tuple $(L, A, P, \Lambda$, $I, C)$. Some of these parameters have already been defined in [8], while others extend the previously proposed model. All of these are defined below.

Hierarchy Levels $(L)$. The notion of hierarchy levels is an extension of our previous model. It is used to determine the relative value of various organizational roles. Thus, one agent is considered to be more valuable (in terms of conversations it commits) than another agent if and only if its hierarchy level is higher than the hierarchy level of the other agent. For each hierarchy level, we define a value range associated with it, i.e $\nu_{\text {range }}=\left[\nu_{\min }(l), \nu_{\max }(l)\right], \forall l \in L$. It is used to define relation between two hierarchy levels. Thus, we will say that one hierarchy level is higher than another hierarchy level if and only if its minimum value is greater than the maximum value of the other hierarchy level, i.e. $l_{1}>l_{2}$ where $l_{1}, l_{2} \in L \Leftrightarrow \nu_{\min }\left(l_{1}\right)>\nu_{\max }\left(l_{2}\right)$.

Agents $(A)$. $A$ indicates the set of communicating agents in organization. As already mentioned, each communicating agent is associated with a hierarchy level, $\forall a \in$ $A, \exists l \in L$ such that $L(a)=l$. The distribution of agents among hierarchy levels determines the type of hierarchical structure in organization. For instance, in pyramidalhierarchical structure, discussed in this paper (see section 4.1), the number of agents in higher hierarchal levels is always smaller than in the lower ones. 
Conversation Protocols $(P) . P$ indicates the set of conversation protocols used in a conversation system. A detailed definition of conversation protocol $p \in P$ can be found in [8]. Here, we refer only to one of its components-a set of its conversation roles, denoted by $R(p)$. For each role, we define the value of its implementation in a given conversation protocol as $\nu(r), \forall r \in R(p)$.

Conversation Topics $(\Lambda)$. $\Lambda$ denotes the set of conversation topics. Each topic has a relative value indicated as $\nu(\lambda), \forall \lambda \in \Lambda$. This value associates each conversation topic to a corresponding hierarchy level, i.e. $\forall \lambda \in \Lambda \exists l \in L$ such that $\nu_{\min }(l) \leq \nu(\lambda) \leq$ $\nu_{\max }(l)$.

Intervals $(I)$. An interval is a time period within the lifetime of a multi-agent system. Thus, we define $I$ as follows: $I=\left\{\left[t_{1}, t_{2}\right] \mid t_{1}, t_{2}\right.$ time stamps, $t_{1} \geq 0, t_{2} \leq$ lifetime, $\left.t_{1} \leq t_{2}\right\}$.

Conversations $(C)$. We define a conversation as a group of agents $g \in 2^{A}$ implementing a conversation protocol $p \in P$ on a conversation topic $\lambda \in \Lambda$ within a time interval $i \in I$. Thus, the $C$ set can be formulated as

$$
C \subseteq\left\{(p, g, \lambda, i) \mid p \in P, g \in 2^{A}, \lambda \in \Lambda, i \in I\right\}
$$

Using this definitions, we can formulate the value of conversation for a certain communicating agent as $\nu(c, a)=\nu(\lambda) \oplus \nu(r)$ where $c=(p, g, \lambda, i), a \in g$ and $r=$ $R(a, c) \in R(p)$. Meaning that the value of conversation $c$ for agent $a$ (participating in it) is a function of conversation topic $\lambda$ and role $r$ (within conversation protocol $p$ ) that agent $a$ implements. The information value of conversations distinguishes between the more important conversations and the less important ones.

A set of conversations $(C)$, generated in hierarchical organizations, has the following characteristics:

- Conversations Distribution. Conversations distribution depends on the distribution of agents among various hierarchy levels. For instance, in pyramidal-hierarchical organizations, lower levels are the "working" levels. Thus, most conversations are held between agents in lower hierarchical levels.

- Conversation Topics. Agents communicate on topics within the scope of their organizational responsibility. Thus, agents mainly communicate on conversation topics associated with their hierarchy level or topics relatively close to it. As a result, agents of higher hierarchy levels commit conversations on more valuable topics.

- Conversation Groups. Agents communicate mostly with their peers, subordinates and their close superiors. Thus, most communications are held between agents of the same hierarchy levels or between agents in relatively close hierarchy levels.

- Conversation Roles. Mostly, agents of higher hierarchical levels implement highervalue roles in conversation protocols.

\subsection{Modelling Overhearing}

In this section, we present our model of overhearing organizations. Section 3.2 introduces overhearing strategies for a single overhearing agent and for teams of overhearers, whereas section 3.2 presents an evaluation technique to compare various overhearing strategies. 
Overhearing Strategies. A single overhearing agent, acting in a cooperative environment, assumes some knowledge on monitored organization. An overhearing agent usually knows what agents communicate in these settings, which protocols are being used, on which topics the conversations are being held and etc. On the other hand, some information remains unknown. For instance, it does not necessarily know the complete list of conversations being held in organization at any given time.

We assume that a single overhearing agent is able to overhear all conversations held by its target agent, i.e. the communicating agent being overheard. Of course, only conversations within overhearing time interval, the time period in which the communicating agent is targeted, are being overheard.

The overhearing agent performs conversation recognition [8] for each conversation. Initially, the overhearing agent does not know the agents, protocol and topic associated with an overheard conversation. Only as the time progresses, the overhearer is able to recognize the various conversation parameters. The overhearing agent starts overhearing assuming that the conversation protocol and topic can be any of the $p \in P$ and $\lambda \in \Lambda$ respectively. Gradually, the overhearer is able to disqualify inappropriate protocols and topics until it determines the correct protocol and topic. This information, at its different stages, can be used to determine whether to continue to overhear the current agent or to find another target.

Since a single overhearing agent can only hear a small subset of conversations in a conversation system, multiple overhearing agents can be deployed to maximize coverage of the overheard conversations. However, the available overhearing resources, i.e. the number of overhearing agents, are limited. Thus, overhearing targets should be carefully chosen in order to increase the total efficiency of overhearing group.

The systematic targeting of communicating agents by an overhearing team is called overhearing strategy. Various strategies can be proposed: centralized vs. distributed, full vs. limited knowledge of conversation systems, various levels of collaboration between overhearing agents, etc.

We focus on centralized overhearing strategies with full information disclosure and leave investigation of other strategies for future work. Here, a central agent has knowledge of the conversation system parameters (e.g. agents' hierarchy levels, conversing agents at time $t$, etc.). Using this information, it directs the choice of target agents for each overhearing agent in overhearing group.

Comparing Overhearing Strategies. Each overhearing strategy may choose to overhear different target agents, and, thus, overhears different conversations. Consequently, some strategies may perform well while others perform poorly. Furthermore, the same overhearing strategy may vary in its performance under different configurations of conversation systems and overhearing resources.

Thus, contrasting overhearing strategies is important in order to determine which strategy should be applied under certain conditions. The overhearing strategies are evaluated in three steps. First, the optimal overhearing value, also referred as optimum, is calculated (Algorithm 1). Optimum, denoted as $\nu_{\text {optimum }}$, is the value of most efficient overhearing possible, i.e. at each time unit $t$ overhearing the communicating agents with highest conversation values. Then, we use Algorithm 2 to calculate the strategy's 
overhearing value, denoted $\nu_{\text {group }}$, which is the accumulative value of all overheard conversations using the specific overhearing strategy. Finally, the overhearing strategy is evaluated as a percentage of optimum, $\left(\nu_{\text {group }} / \nu_{\text {optimum }}\right) * 100$. Using this evaluation, we compare various overhearing strategies.

Algorithm 1 introduces the calculation of optimal overhearing value. For each time unit $t$ (lines 2-8), optimum at time $t$ is calculated and accumulated in $\nu_{\text {optimum }}$ (line 7). The optimum at time $t$ for $k$ overhearing agents is defined as a sum of conversation values of $k$ agents with the highest conversation values at time $t$ (lines 6-7). A conversation value of agent $a \in A$ at time $t$-denoted as $\nu_{t}(a)$-is the accumulative value of its conversations at time $t$ (lines 4-5). This algorithm makes a simplifying assumption on changing overhearing targets. It assumes that a change of overhearing target by an overhearing agent is instantaneous and has no cost. This assumption is also used in other calculations.

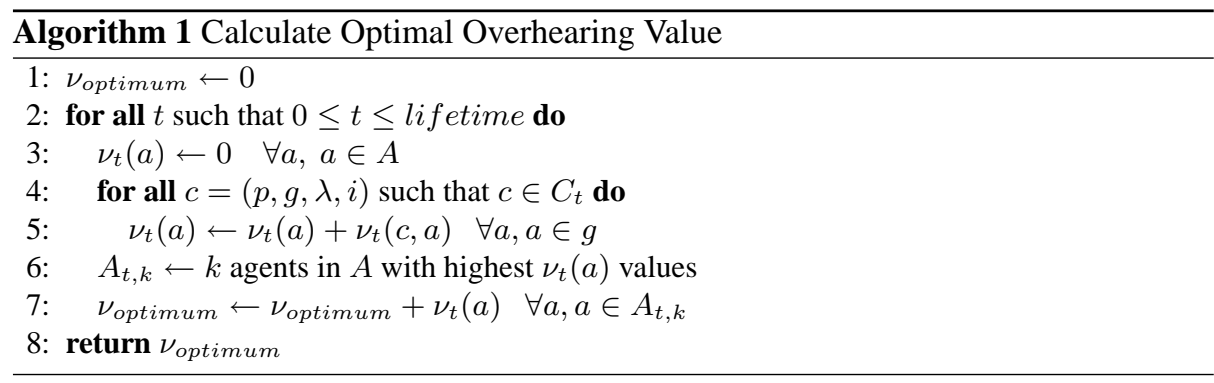

Algorithm 2 presents the calculation of overhearing value for a team of $k$ overhearers implementing specific strategy. Again, for each time unit $t$ (lines 2-6), we calculate its overhearing value at time $t$ and accumulate it in $\nu_{\text {group }}$ (line 6). An overhearing value at time $t$ is defined as an accumulative conversation value of overheard agents. Thus, in lines 3-6, for each overheard conversation, in a set of overheard conversations at time $t$ $\left(O C_{t}\right)$, its conversation value is accumulated for each participating agent that has been overheard (the $O A_{t}$ parameter indicates the set of agents overheard at time $t$ ).

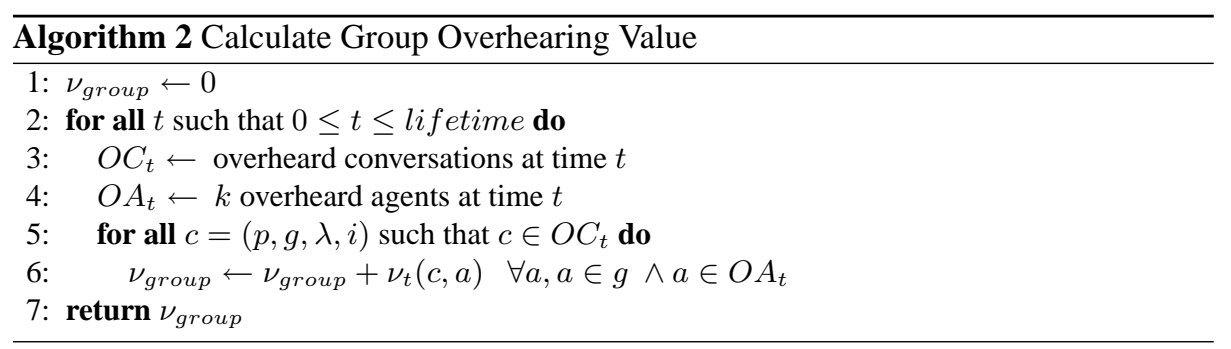




\section{Experiments}

This section presents an empirical analysis of limited-resource overhearing in pyramidalhierarchical organizations. Section 4.1 defines the experimental settings, while Section 4.2 analyzes the results of the experiments.

\subsection{Experimental Settings}

The experimental settings have been defined to simulate communications in pyramidalhierarchical organizations. The number of communicating agents, i.e. $|A|$, was set to 50 simulating relatively small organizations. These simulated settings have been defined as highly hierarchical: the number of hierarchy levels $(|L|)$ was set to 7 . The value range for each hierarchy level was calculated as a relative portion of [1,100], which was divided equally $\forall l \in L$. Agents were hyperbolically distributed among different hierarchy levels to simulate pyramidal-hierarchical structure. Thus, the number of agents, related to a hierarchy level, becomes smaller as hierarchy levels get higher.

The number of topics, i.e. $|\Lambda|$, has been set to 80 . This value reflects our intuition that each agent has at least one conversation topic under its direct responsibility. The additional topics are generally common to all communicating agents. Each topic has been randomly given a value between 1 and 100 associating it with a hierarchy level.

The number of protocols was defined as 25 , almost twice the number of interaction protocols specified by FIPA [10], simulating a diversity of interactions that are possible in organization. The duration of each protocol has been randomly set to a value within $\{5,10,15,20,25\}$, whereas the lifetime of entire conversation system was determined to 1000 . For each protocol, two roles have been defined. Their values were randomly set to one of the following combinations: $\{50,50\},\{67,33\},\{75,25\}$ and $\{99,1\}$. In this manner, we simulate differences in the importance of roles within the conversation. Finally, the conversation value is calculated using an accumulative function, i.e. $\nu(c, a)=\nu(\lambda)+\nu(r)$ (see Section 3.1). Thus, conversation values range from 1 to 199 .

In the experiments below, we generated conversation systems and simulated their dynamic execution. These conversation systems have been statistically generated supporting the characteristics of hierarchical organizations described in Section 3.1. In each conversation system, a constant level of conversations was maintained at all time throughout the lifetime of the conversation system.

Addressing certain configuration, various overhearing strategies can be compared as shown in Section 3.2. In Section 4.2, we compare proposed overhearing strategies using their evaluation values (as a percentage of optimum) in different configurations of activity levels, overhearing resources and value of information. Each evaluation is performed based on an average of 50 independent experiments committed with respect to corresponding configuration.

\subsection{Results}

Centralistic Overhearing Strategies. Our initial hypothesis has been that the most successful overhearing in pyramidal-hierarchical organizations (under the restriction of selectivity) can be achieved by overhearing conversations committed by most important 
agents. The main intuition behind this hypothesis is that most important agents commit the most valuable conversations.

Thus, our first overhearing strategy, called MostImportantStatic, was defined to implement this hypothesis. According to this strategy, $k$ overhearing agents were set to overhear the $k$ most important agents (in terms of their hierarchy level). To examine our argument, we defined an additional overhearing strategy, called RandomStatic, to serve as a control strategy. Here, $k$ overhearers were set to target $k$ random agents chosen at the beginning of the experiment.

The comparison of these two strategies is presented in Figure 1. The values on the $\mathrm{X}$-axe show the activity levels of the examined conversation systems, i.e. the ratio between the number of conversations at time $t\left(\left|C_{t}\right|\right)$ and the number of communicating agents $(|A|)$, whereas the Y-axe determines the performance of compared strategies as a percentage of optimum. In results shown in Figure 1, the overhearing coverage, defined as the ratio between the number of overhearers and the number of communicating agents $-k /|A|$, was set to $30 \%$. We can clearly see that MostImportantStatic strategy has been more efficient. However, both strategies perform poorly for low and medium activity levels (1\%-100\%)—maximum up to $70 \%$ and $40 \%$ of optimum respectively.

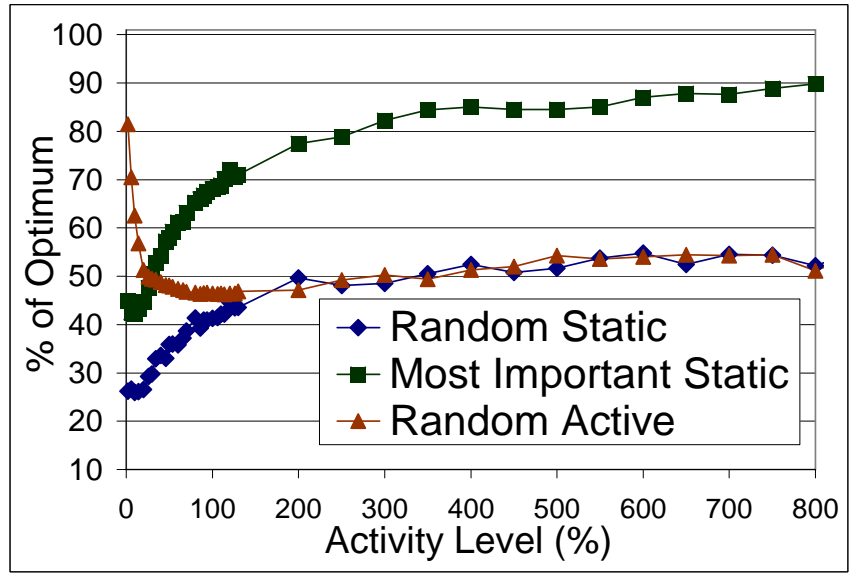

Fig. 1. Initial Overhearing Strategies

Analyzing this poor performance, we came to the conclusion that the main drawback of these strategies is that their overhearing targets are determined statically. Thus, in case that the overheard agent is idle (i.e. committing no conversations), overhearing it has zero-value. In low and medium activity levels, the probability of an agent to be idle is relatively high. In such conditions, the static strategies perform poorly. However, as the activity level grows, the probability of an agent to be idle reduces. Thus, static overhearing strategies monotonically rise as the activity level grows until the probability of an agent to be idle is close (or equal) to 0 .

We developed a new overhearing strategy based on this conclusion. Similarly to RandomStatic strategy, RandomActive chooses $k$ target agents at the beginning of the experiment. However, each time a target agent is idle, an alternative target is randomly 
chosen. Figure 1 shows also the performance results of this strategy. Here, we can see that at low activity levels RandomActive performs better than the MostImportantStatic strategy.

Based on the insight gained, we develop two additional strategies. The first strategy is called MostImportantActive strategy. It improves our initial MostImportantStatic strategy. The overhearing targets are determined as the $k$ most important agents from those that are currently active. The second overhearing strategy, called MostActive, implements a slightly different approach. In contrast to MostImportantActive, MostActive targets $k$ most active agents, i.e. $k$ agents that commit the highest number of conversation at time $t$. Since the overhearing agent overhears all conversations committed by its target, overhearer can be efficient due to quantity of overheard conversations and not their "quality". Moreover, in pyramidal-hierarchical organizations, most conversations are held between agents of lower hierarchy levels. Thus, in fact, MostActive targets the less important agents. Both overhearing strategies proved to be highly efficient (Figure 2). Thus, the rest of the paper will focus on a detailed comparison of these two strategies.

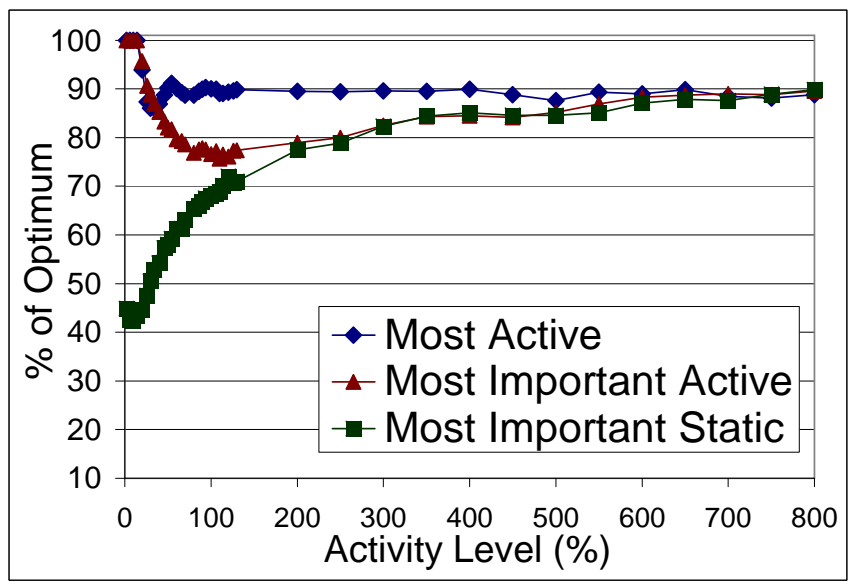

Fig. 2. Most Important vs. Most Active

Most Important Active vs. Most Active. At first, we analyzed these two strategies in various conditions of activity level maintaining the overhearing resources constant. This comparison led to a surprising result. The MostImportantActive strategy behaves as a parabolic curve with a long tail as the activity level increases, while MostActive strategy remains linear (see Figures 2, 3 and 4 for example).

This result can be explained as follows. At low activity levels, each communicating agent is either idle or involved in few conversations. Thus, overhearing important active agents is more efficient due to the higher value of their conversations. However, as the activity level grows, low-level agents become involved in more and more conversations. Consequently, at some point, overhearing a number of less-valuable conversations, committed by less important agents, becomes more efficient than overhearing a single high-value conversation of an important agent. The efficiency gap, between these 
strategies, becomes more significant as activity level increases and low-level agents become involved in greater number of conversations. However, at some point, the efficiency trade-off between these strategies changes. The conversation activity of highlevel agents increases as well. At some point, it again becomes more efficient to overhear few conversations committed by important agents than to overhear many conversations held by low-level agents.

This surprising result implies that in monitoring organizations, an overhearer should decide when it is more efficient to target few highly-valuable communications, and when the total information, gathered from a large number of less-valuable communications, is more efficient in understanding the current status of the organization.

Value of Conversations. To understand the nature of these two strategies, we sought to isolate the parameters influencing the intersection points. As already explained above, this trade-off depends on whether it is more efficient to target small number of highvalue communications or a large number of low-value conversations. Therefore, in the following experiments we changed the ratio between the low-value and the high-value conversations, i.e. the ratio between an average value of conversations in high and low hierarchy levels respectively.

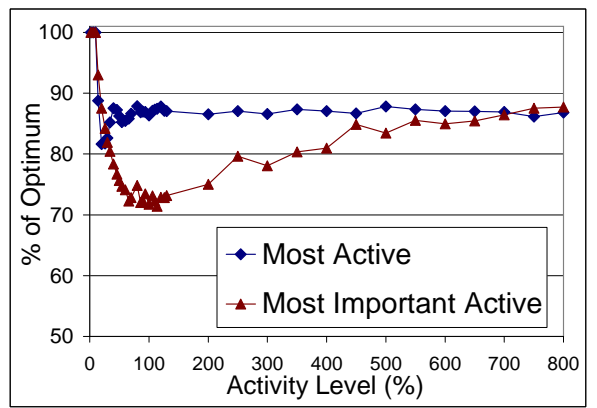

(a) Conversations Value Ratio 1:3

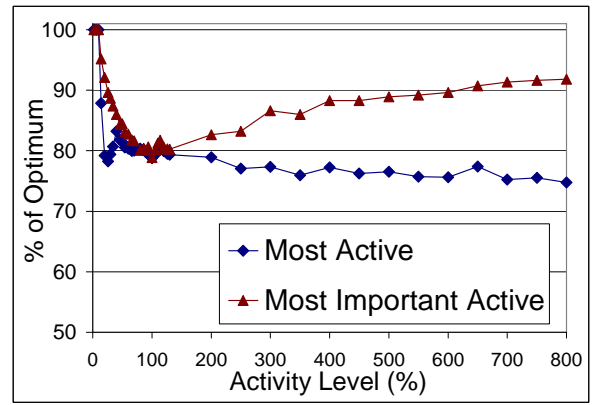

(c) Conversations Value Ratio 1:5

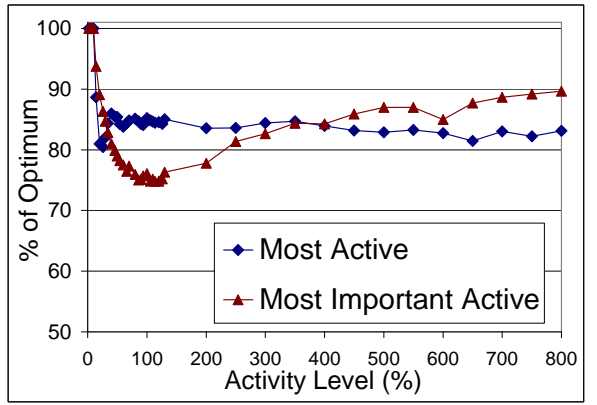

(b) Conversations Value Ratio 1:3 $\frac{1}{2}$

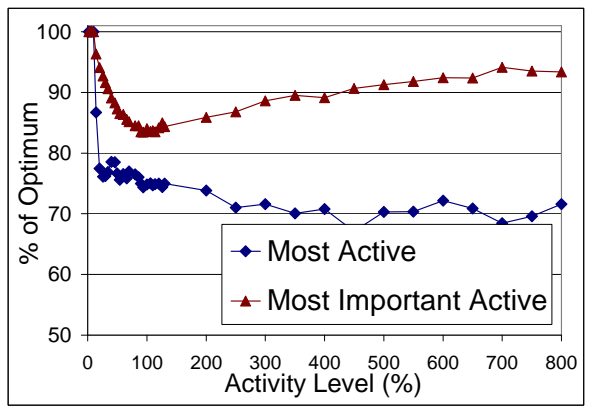

(d) Conversations Value Ratio 1:8

Fig. 3. Overhearing Strategies Comparison with Respect to Conversations Value.

In our experimental environment, the value of conversation ranges between 1 and 199. In average, conversations committed by agents of lowest hierarchy level are valued nearly 50, while conversations of highest-level agents value around 150 (ratio 1:3). In 
our experiments, we have also examined the behavior of the proposed strategies for additional ratios.

Figures 3(a-d) show the performance results of these strategies for 1:3, 1:3 $\frac{1}{2}, 1: 5$ and 1:8 ratios (where overhearing coverage is set to $20 \%$ ). It can clearly be seen that as the ratio of conversations value increases, the MostImportantActive strategy improves, while the MostActive strategy deteriorates. At some point (Figure 3-c), the two intersection points turn into one, i.e. the two strategies intersect at the bottom of the parabola. Then, in Figure 3-d, the two graphs do not intersect at all-the MostImportantActive strategy remains more efficient even in its parabolic form.

Thus, in case the difference between high-level and low-level conversation values is significant, it is more efficient to target highly important agents than to overhear lowlevel, highly-communicative ones.

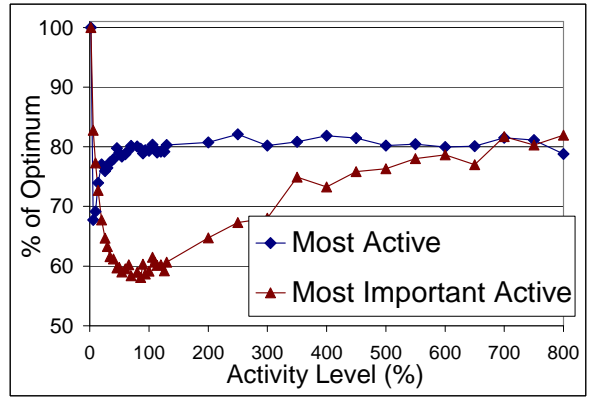

(a) Overhearing Coverage 5\%

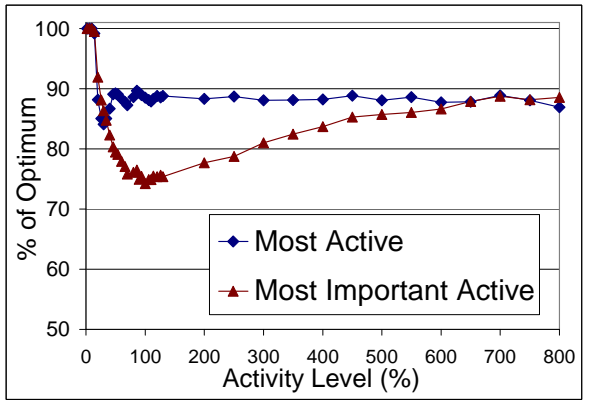

(b) Overhearing Coverage $25 \%$

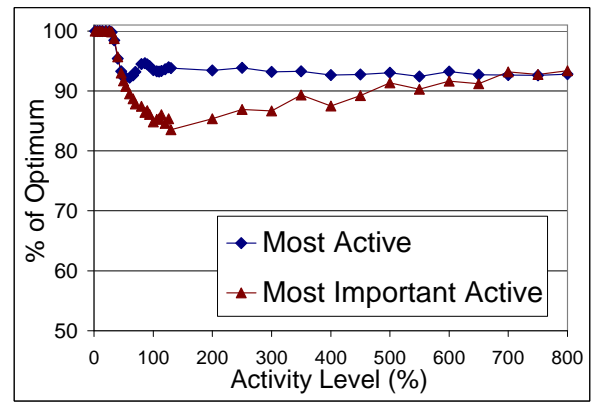

(c) Overhearing Coverage $50 \%$

Fig. 4. Overhearing Strategies Comparison with Respect to Overhearers Number.

Number of Overhearers. Finally, we examine the influence of selectivity. Figures 4(ac) show the behavior of the proposed strategies for various levels of overhearing coverage $(5 \%, 25 \%$ and $50 \%)$. We can see that both strategies become more efficient with higher overhearing coverage. Clearly, this conclusion is to some extent straightforward. However, an additional, less-trivial conclusion can be made. The gap between the MostActive and the MostImportantActive strategies becomes less significant in larger 
overhearing groups. It can be seen that the parabolic curve of MostImportantActive graph becomes less concave. In large overhearing groups, this effect can be explained by a significant overlap in overhearing targets for both strategies.

\section{Conclusions \& Future Work}

Lately, overhearing has become an acceptable method for monitoring multi-agent systems. Previous investigations proposed an extensive set of techniques and practises using overhearing. However, the problem of selective overhearing, under the restriction of limited overhearing resources, has not been addressed so far.

In this paper, we present an empirical study of limited-resource overhearing for hierarchically-structured organizations. Our work provides a model addressing both the characteristics of conversations in such organizations and the overhearing strategies appropriate for these settings.

Based on this model, we performed a set of experiments simulating conversations in hierarchical organizations and examined some of the proposed overhearing strategies. Analyzing the results of performed experiments, we were able to determine efficient overhearing strategies and to isolate the parameters influencing their performance. The main conclusions of our experiments can be summarized as:

1. Efficient overhearing strategies. The selective overhearing strategies of targeting $k$ most important active agents and of targeting $k$ most communicative agents have been found to be highly efficient.

2. Strategies Comparison. A double efficiency trade-off has been found comparing these two strategies. Thus, in some conditions, it is more efficient to overhear few highly-valuable communications, while, in other conditions, it is more efficient to target large number of less-valuable conversations.

3. Value of information. In organizations, where conversations committed by agents in high hierarchy levels are considerably more important than conversations committed in low hierarchy levels, the strategy of targeting $k$ most important agents achieves better performance results.

4. Number of overhearers. For larger overhearing teams, both strategies perform better and the performance gap between them decreases due to the significant overlap in overheard conversations.

Currently, only centralized overhearing strategies have been concerned. Thus, in the future, we would like to examine the behavior of distributed and other overhearing strategies in these settings.

\section{References}

1. Kaminka, G., Pynadath, D., Tambe, M.: Monitoring teams by overhearing: A multi-agent plan-recognition approach. JAIR 17 (2002) 83-135

2. Novick, D., Ward, K.: Mutual beliefs of multiple conversants: A computational model of collaboration in air traffic control. In: Proceedings of AAAI-93. (1993) 196-201 
3. Aiello, M., Busetta, P., Dona, A., Serafini, L.: Ontological overhearing. In: Proceedings of ATAL-01. (2001)

4. Busetta, P., Dona, A., Nori, M.: Channelled multicast for group communications. In: Proceedings of AAMAS-02. (2002)

5. Busetta, P., Serafini, L., Singh, D., Zini, F.: Extending multi-agent cooperation by overhearing. In: Proceedings of CoopIS-01. (2001)

6. Legras, F.: Using overhearing for local group formation. In: Proceedings of AAMAS-02. (2002)

7. Rossi, S., Busetta, P.: Towards monitoring of group interactions and social roles via overhearing. In: Proceedings of CIA-04, Erfurt, Germany (2004) 47-61

8. Gutnik, G., Kaminka, G.: Towards a formal approach to overhearing: Algorithms for conversation identification. In: Proceedings of AAMAS-04. (2004)

9. Platon, E., Sabouret, N., Honiden, S.: T-compound: An agent-specific design pattern and its environment. In: Proceedings of 3rd international workshop on Agent Oriented Methodologies at OOPSLA 2004. (2004) 63-74

10. FIPA: Fipa-ACL specifications, at www.fipa.org (2005) 